Lost in translation

The culture of academia needs to change if scientists are to bridge the gap between research and the development of drugs and vaccines for neglected diseases in the developing world, says Declan Butler.

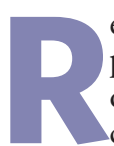

ead any scientific paper or grant proposal on basic research into neglected diseases and you inevitably find a claim that the work could lead to new therapies for diseases that affect millions of people in the developing world. Few, in fact, ever do, and scientists, universities and research funders are waking up to the fact that part of the blame lies with them and their perpetuation of a reward culture that focuses excessively on papers and patents, and not on whether the research actually benefits society.

In disease research, academia has traditionally restricted its role to basic research. Subsequent development is then left to the pharmaceutical industry. But when it comes to neglected diseases - those that disproportionally affect poor and marginalized populations - the drugs and vaccines have low returns, so commercial firms cannot fork out for the expensive development. As a result, there is a 'translational gap' in which promising research leads sit on the shelf, and potential drugs and vaccines go undeveloped.

The academic reward culture means that researchers have little incentive to do the 'grunt work' needed to close this gap because it does not generate papers in top jour-

"Having a chemical
structure that kills
your parasite is only
one of many aspects
of what makes a
drug a drug."
$\quad$ - Els Torreele
engagement of academics in the development of new drugs. Over the past decade, the translational gap has started to close for neglected diseases, thanks to the emergence of a series of public-private partnerships between charities, large pharmaceutical companies and small biotech firms. These partnerships are run like businesses, but not for profit, and include the Malaria Vaccine Initiative in Bethesda, Maryland; the Medicines for Malaria Venture in Geneva, Switzerland; the Global Alliance for TB Drug Development; and the Drugs for Neglected Diseases Initiative (DNDi) in Geneva, Switzerland, which is seeking cures for the 'most neglected' diseases, such as sleeping sicknals, says Mel Spigelman, director of research and development at the Global Alliance for TB Drug Development in New York. The competitive nature of academic research and of the publication reward system can also lead to unnecessary duplication of work, and hinders the sharing of data, he adds. ness (African trypanosomiasis), Chagas' disease and leishmaniasis.

Although only 13 of the 1,223 drugs developed since 1975 have been for neglected diseases, dozens are now in the pipeline of public-private partnerships. These partnerships also play a key part in helping academics to tailor their research to the needs of drug development by creating focused projects that bring them into contact with industry scientists (see pages 164 and 166).

That cross-fertilization is important because translational research requires skills and a culture that universities typically lack, says Victoria Hale, chief executive of the non-profit drug company the Institute for OneWorld Health in San Francisco, California, which is developing drugs for visceral leishmaniasis, malaria and Chagas' disease. Academic institutions are often naive about what it takes to develop a drug, she says, and much basic research is therefore unusable. That's because few universities are willing to support the medicinal chemistry research needed to verify from the outset that a compound will not be a dead end in terms of drug development.

Academics will currently publish, say, a chemical scaffold, which they bill as a potential new target for parasites. "But had a medicinal chemist looked at it, he might immediately see that it will never work as a drug, because it has an inappropriate solubility or toxicological profile," says Els Torreele, a product manager at the DNDi. "Having a chemical structure that 
kills your parasite is only one of many aspects of what makes a drug a drug."

Ted Bianco, director of technology transfer at the Wellcome Trust in London, agrees. "It's fine if a researcher is just using a compound as a ligand to probe a biological process," he says, "but don't kid yourself it's a drug unless you ask whether it has druggable properties." What's needed, says Hale, is a 'target product profile', which sets out the appropriate drug chemistry properties. "Getting a drug through regulatory processes is not just about how good your science is and how great your trials are; it is much more complex," says Hale. "And aca-

"Non-patenting or
free licensing might
be the best way
to make sure that
research is used for
public benefit."
- Carol Mimura

streams. The thicket of resultant patents has meant that groups seeking to develop drugs and vaccines for neglected diseases simply cannot afford to do the necessary research.

Although universities with strong tropicaldisease research centres are often well aware of the need to make patents freely available, many techniques needed in drug development, such as drug stabilization, are patented by universities that have no interest in tropical diseases. Persuading a university to let organizations such as the DNDi use a technology freely often takes more than a year, and almost always requires intervention at the highest levdemics don't have the experience - they need to hire people from the drug industry."

\section{Seeds of success}

To tackle this problem, the Wellcome Trust has started a £91-million (US\$182-million) five-year Seeding Drug Discovery initiative for neglected diseases, which aims to provide universities with the funds to do the sort of screening and medicinal chemistry usually found only in industry. The initiative includes an $£ 8.1$-million award to the University of Dundee, Scotland, which is working with the DNDi on drugs for the most neglected diseases.

But universities could also play a part by engaging actively in development research for neglected diseases, says Hale. Over the past two decades, academic institutions have been putting greater emphasis on wealth creation. Universities will often patent everything they can in the hope of generating additional revenue

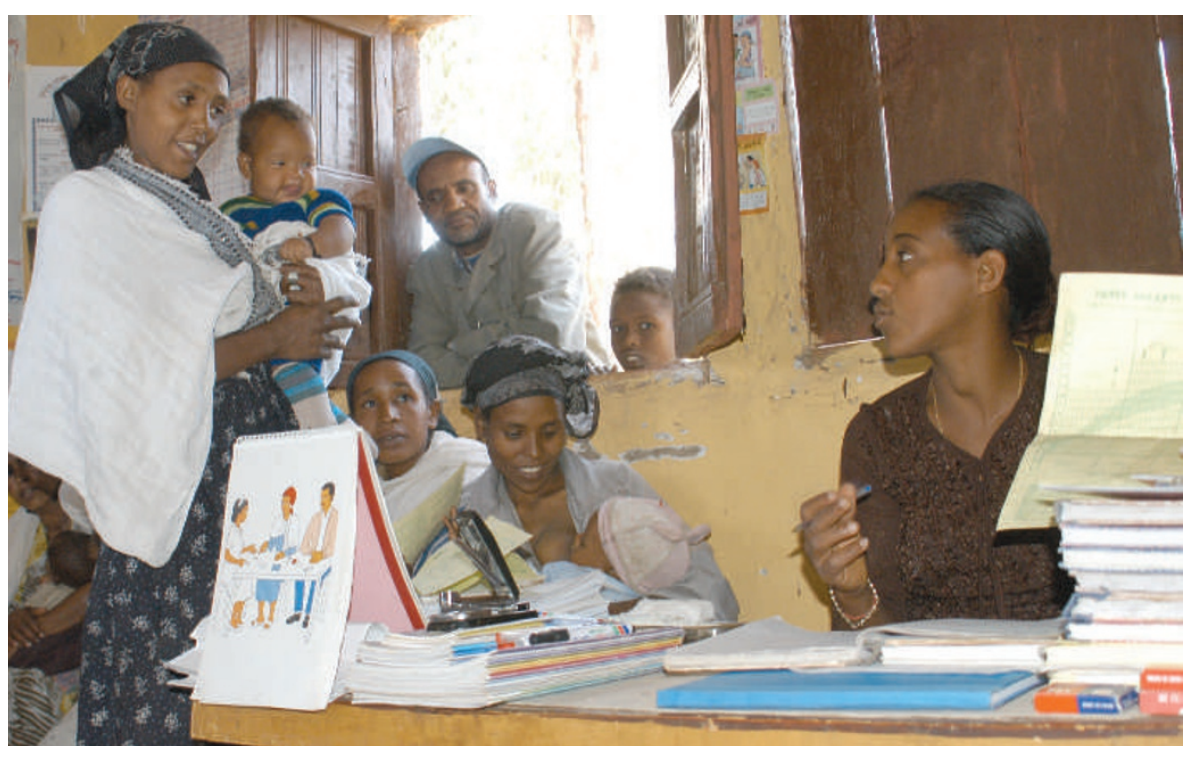

Vaccination programmes in countries such as Ethiopia depend on having affordable products. projects, including a cheaper artemisinin drug for malaria, and a possible vaccine for tuberculosis (see page 174).

Harris provided a moral compass that led to a cultural shift at Berkeley, says Mimura. "Organizations perform to what they are measured by. Traditional metrics such as the numbers of patents, licences signed and amount of licensing revenue, seem to be the sole goals that a technology-transfer office aspires to," she says, "when in fact non-patenting or free licensing might be the best way to make sure that research is used for public benefit."

Although it is more difficult to measure, Berkeley now treats the social impact of its intellectual property as an equal or more important bottom line than economic gain, says Mimura. She thinks that the university will gain in the long term by attracting donations and philanthropic partnerships as a result of the reputation and goodwill generated. "We need to manage our intellectual property with an eye towards the ultimate ripple effect of its management, not just the short-term outcomes," she says.

\section{Pressure tactics}

In 2001, Yale University and Bristol-Myers Squibb agreed to allow South Africa to make generic versions of an AIDS drug, d4T (stavudine), which Yale had licensed to the company. Pressure has also come from a student group based in Philadelphia called Universities Allied for Essential Medicines, which last November released the Philadelphia Consensus Statement, which called on universities to adopt policies in favour of neglected diseases.

And the momentum is growing. The US National Institutes of Health has adopted similar progressive policies to those of Berkeley, as have several universities in North America. And in March, a dozen universities in the United States issued a joint statement pledging to be "mindful of their primary mission to use patents to promote technology development for the benefit of society", including making provisions for therapeutics, diagnostics and agricultural technologies in developing countries (see http://tinyurl.com/2qmtr4).

But if the pendulum is swinging back from an emphasis on wealth creation to one on social good in the United States, the same is not true everywhere, says Torreele. Ironically, it is now often easier to get royalty-free patents from a US university than it is from some in India, Brazil or Thailand, she says, as emerging economies follow the West's lead in emphasizing wealth creation from academic research.

Declan Butler is Nature's European correspondent. 